\title{
Well-posedness of a Debye type system endowed with a full wave equation.
}

\author{
Arnaud Heibig ${ }^{1}$ \\ Université de Lyon, Institut Camille Jordan, INSA-Lyon, Bât. Leonard de Vinci No. 401, 21 Avenue Jean \\ Capelle, F-69621, Villeurbanne, France.
}

\begin{abstract}
We prove well-posedness for a transport-diffusion problem coupled with a wave equation for the potential. We assume that the initial data are small. A bilinear form in the spirit of Kato's proof for the Navier-Stokes equations is used, coupled with suitable estimates in Chemin-Lerner spaces. In the one dimensional case, we get well-posedness for arbitrarily large initial data by using Gagliardo-Nirenberg inequalities.
\end{abstract}

Keywords: Transport-diffusion equation, wave equation, Debye system, Chemin-Lerner spaces, Gagliardo-Nirenberg inequalities.

\section{Introduction.}

Transport-diffusion equations have a vast phenomenology and have been widely studied. See, among others, [2], [3], [7], [10] in the case of the semi-conductor theory, and [5] in the case of Fokker-Planck equations. The goal of this note is to prove existence and uniqueness of the solution for a modify semi-conductor equation.

In order to simplify the presentation, we restrict to the case of a single electrical charge. The novelty of our equations is that we replace the Poisson equation on the potential by a wave equation. This is a quite natural change, since the electric charge itself depends on the time. From a mathematical point of view, switching from a Poisson equation to a wave equation roughly amounts to the loss of one derivative in the estimates on the potential. Moreover, it seems that one is bound to work in $L_{t}^{p}$ spaces with $1 \leq p \leq 2$ due to the usual Strichartz estimates.

In this paper, we prove the existence of a mild solution in Chemin-Lerner spaces $\tilde{L}^{1}(0, T$, $\left.\dot{H}^{n / 2-1}\left(\mathbb{R}^{n}\right)\right)$. We first restrict to the case of small initial data $(n \geq 2)$, and use a variant of the Picard fixed point theorem as in the proof of Kato's and Chemin's theorems for the Navier-Stokes (and related) equations. See [9], [6], [4] and also [8], [1]. In particular, we work in homogeneous Sobolev spaces in order to get $T$-independent estimates for the heat equation. Note also that our bilinear form depends on a nonlocal term, given as the solution of the wave equation on the potential.

In the case $n=1$, well posedness is established for arbitrary large initial data (section 4). Local well posedness is obtained as in section 3. The global existence is proved by combining the usual $L^{1}$ estimate with a Gagliardo-Nirenberg inequality, in the spirit of [3].

\footnotetext{
${ }^{1}$ Corresponding author. E-mail adress: arnaud.heibig@insa-lyon.fr Fax: +33 472438529
} 


\section{Equations and preliminary results.}

We begin with some notations. In this section $n \geq 2, T>0$, and $s<n / 2$ are given. The homogeneous Sobolev spaces $\dot{H}^{s}\left(\mathbb{R}^{n}\right)$ are often denoted by $\dot{H}^{s}$. For $p \geq 1$, we also use the CheminLerner spaces $\tilde{L}^{p}\left(0, T, \dot{H}^{s}\left(\mathbb{R}^{n}\right)\right)=\tilde{L}^{p}\left(0, T, \dot{B}_{2,2}^{s}\left(\mathbb{R}^{n}\right)\right)$, or simply $\tilde{L}_{T}^{p}\left(\dot{H}^{s}\right)$. Recall that a distribution $f \in \mathscr{S}^{\prime}(] 0, T\left[\times \mathbb{R}^{n}\right)$ belongs to the space $\tilde{L}_{T}^{p}\left(\dot{H}^{s}\right)$ iff $\dot{S}_{j} f \rightarrow 0$ in $\mathscr{S}^{\prime}$ for $j \rightarrow-\infty$, and $\|f\|_{\tilde{L}_{T}^{p}\left(\dot{H}^{s}\right)}:=$ $\left\|\left(2^{j s}\left\|\dot{\Delta}_{j} f\right\|_{L_{T}^{p}\left(L^{2}\right)}\right)_{j \in \mathbb{Z}}\right\|_{l^{2}(\mathbb{Z})}<\infty$. Here, $\dot{S}_{j} f$ and $\dot{\Delta}_{j} f$ are respectively the low frequency cut-off and the homogeneous dyadic block defined by the usual Paley-Littlewood decomposition. See [1] p.98 for details. Last, we write $\nabla$ for the (spatial) gradient, div for the divergence and $\Delta=\operatorname{div} \nabla$.

We now give the equations we are dealing with. Set $s=n / 2-1$. Consider the Cauchy problem on the scalar valued functions $u$ and $V$ defined on $\mathbb{R}_{+} \times \mathbb{R}_{x}^{n}$

$$
\begin{aligned}
& \partial_{t} u-\Delta u=\operatorname{div}(u \nabla V) \\
& \partial_{t t} V-\Delta V=u \\
& u(0)=u_{0} \\
& V(0)=V_{0}, V_{t}(0)=V_{1}
\end{aligned}
$$

For $u_{0} \in \dot{H}^{s},\left(\nabla V_{0}, V_{1}\right) \in \dot{H}^{s} \times \dot{H}^{s}$ and $u \in \tilde{L}_{T}^{1}\left(\dot{H}^{s}\right)$ given, we denote by $S\left(u, V_{0}, V_{1}\right) \in C^{0}\left(0, T, \mathscr{S}^{\prime}\left(\mathbb{R}^{n}\right)\right)$ the unique solution of the wave equation 2, 4. With these notations, the system 11-4 is interpreted as the following problem $(\mathrm{P})$ :

find $u \in \tilde{L}_{T}^{1}\left(\dot{H}^{s}\right)$ such that

$$
\begin{aligned}
& \partial_{t} u-\Delta u=\operatorname{div}\left(u \nabla S\left(u, V_{0}, V_{1}\right)\right) \\
& u(0)=u_{0}
\end{aligned}
$$

For future reference, we recall a standard result on the heat equation (see [1] p.157)

Proposition 2.1. Let $T>0, \sigma \in \mathbb{R}^{n}$ and $1 \leq p \leq \infty$. Assume that $u_{0} \in \dot{H}^{\sigma}$ and $f \in \tilde{L}_{T}^{p}\left(\dot{H}^{\sigma-2+\frac{2}{p}}\right)$. Then the problem

$$
\begin{aligned}
& \partial_{t} u-\Delta u=f \\
& u(0)=u_{0}
\end{aligned}
$$

admits a unique solution $u \in \tilde{L}_{T}^{p}\left(\dot{H}^{\sigma+\frac{2}{p}}\right) \cap \tilde{L}_{T}^{\infty}\left(\dot{H}^{\sigma}\right)$ and there exists $C>0$ independent of $T$ such that, for any $q \in[p, \infty]$

$$
\|u\|_{\tilde{L}_{T}^{q}\left(\dot{H}^{\left.\sigma+\frac{2}{q}\right)}\right.} \leq C\left(\|f\|_{\tilde{L}_{T}^{p}\left(\dot{H}^{\sigma-2+\frac{2}{p}}\right)}+\left\|u_{0}\right\|_{\dot{H}^{\sigma}}\right)
$$

Moreover, for $f=0$, we have $u \in C^{0}\left([0, T], \dot{H}^{\sigma}\right) \hookrightarrow L^{1}\left([0, T], \dot{H}^{\sigma}\right)$.

The same statements hold true in nonhomogeneous Sobolev spaces with a constant $C=C_{T}$ depending on $T$.

In the sequel, we denote the solution $u$ of proposition 2.1 by

$$
u(t)=e^{t \Delta} u_{0}+\int_{0}^{t} e^{(t-\tau) \Delta} f(\tau) d \tau
$$

We will prove an existence result for problem $(\mathrm{P})$ by combining proposition 2.1 with the following $\dot{H}^{s}$ estimate for the solution $S\left(u, V_{0}, V_{1}\right)$ of the wave equation (see [1] pp. 360-361)

$$
\left\|\nabla S\left(u, V_{0}, V_{1}\right)\right\|_{\tilde{L}_{T}^{\infty}\left(\dot{H}^{s}\right)} \leq C\left(\left\|\nabla V_{0}\right\|_{\dot{H}^{s}}+\left\|V_{1}\right\|_{\dot{H}^{s}}+\|u\|_{\tilde{L}_{T}^{(}\left(\dot{H}^{s}\right)}\right)
$$




\section{Existence and uniqueness in the case $n \geq 2$.}

This part is devoted to the proof of existence of a mild solution to problem $(\mathrm{P})$.

Theorem 3.1. Let $n \geq 2$ and $s=n / 2-1$. There exists $\eta>0$ such that, for any $T>0, u_{0} \in \dot{H}^{s-2}$, $\left(\nabla V_{0}, V_{1}\right) \in \dot{H}^{s} \times \dot{H}^{s}$ with

$$
\left\|u_{0}\right\|_{\dot{H}^{s-2}}+\left\|\nabla V_{0}\right\|_{\dot{H}^{s}}+\left\|V_{1}\right\|_{\dot{H}^{s}} \leq \eta
$$

there exists exactly one solution to the problem

find $u \in \tilde{L}_{T}^{1}\left(\dot{H}^{s}\right)$ such that

$$
u(t)=e^{t \Delta} u_{0}+\int_{0}^{t} e^{(t-\tau) \Delta} d i v\left(u \nabla S\left(u, V_{0}, V_{1}\right)\right)(\tau) d \tau
$$

When the above assumptions are replaced by $u_{0} \in \dot{H}^{s}$ and $\left\|\nabla V_{0}\right\|_{\dot{H}^{s}}+\left\|V_{1}\right\|_{\dot{H}^{s}} \leq \eta$ small enough, we get local in time existence and uniqueness.

We will use the following classical lemma (see for instance [1] p.357). In this lemma, $\bar{B}(0, r) \subset E$ denotes the closed ball of center 0 and radius $r>0$.

Lemma 3.1. Let $E$ be a Banach space. Let $\mathscr{B}: E \times E \rightarrow E$ be a continuous bilinear map and $\mathscr{L}: E \rightarrow E$ be a linear continuous map with $\|\mathscr{L}\|<1$. Let $0<\alpha<(1-\|\mathscr{L}\|)^{2} /(4\|\mathscr{B}\|)$. Then, for any $\gamma \in \bar{B}(0, \alpha)$, there exists exactly one $x \in \bar{B}(0,2 \alpha)$ such that $x=\gamma+\mathscr{L}(x)+\mathscr{B}(x, x)$.

In order to use lemma [3.1, for $T>0$ and $\left(\nabla V_{0}, V_{1}\right) \in \dot{H}^{s} \times \dot{H}^{s}$ given, set $E_{T}=\tilde{L}_{T}^{1}\left(\dot{H}^{s}\right)$ and define $\mathscr{B}_{T}: E_{T} \times E_{T} \rightarrow E_{T}$ by

$$
\mathscr{B}_{T}(u, w)=\int_{0}^{t} e^{(t-\tau) \Delta} \operatorname{div}(u \nabla S(w, 0,0))(\tau) d \tau
$$

We also define $\mathscr{L}_{T}: E_{T} \rightarrow E_{T}$ by

$$
\mathscr{L}_{T}(u)=\int_{0}^{t} e^{(t-\tau) \Delta} \operatorname{div}\left(u \nabla S\left(0, V_{0}, V_{1}\right)\right)(\tau) d \tau
$$

Theorem 3.1 is an immediate consequence of lemma 3.1 and the following $T$-independent estimates.

Lemma 3.2. Let $n \geq 2$ and $s=n / 2-1, u_{0} \in \dot{H}^{s-2},\left(\nabla V_{0}, V_{1}\right) \in \dot{H}^{s} \times \dot{H}^{s}$. Then, there exists $C_{i}>0(0 \leq i \leq 2)$ such that, for any $T>0$ and any $u \in E_{T}, w \in E_{T}$, we have

$$
\begin{aligned}
& \left\|\mathscr{B}_{T}(u, w)\right\|_{E_{T}} \leq C_{0}\|u\|_{E_{T}}\|w\|_{E_{T}} \\
& \left\|\mathscr{L}_{T}(u)\right\|_{E_{T}} \leq C_{1}\left(\left\|\nabla V_{0}\right\|_{\dot{H}^{s}}+\left\|V_{1}\right\|_{\dot{H}^{s}}\right)\|u\|_{E_{T}} \\
& \left\|e^{t \Delta} u_{0}\right\|_{E_{T}} \leq C_{2}\left\|u_{0}\right\|_{\dot{H}^{s-2}}
\end{aligned}
$$

Proof. Inequality 16 follows from proposition 2.1. Inequalities 14 and 15, amounts to

$$
\left\|\mathscr{B}_{T}(u, w)+\mathscr{L}_{T}(u)\right\|_{\tilde{L}_{T}^{1}\left(\dot{H}^{s}\right)} \leq C\|u\|_{\tilde{L}_{T}^{1}\left(\dot{H}^{s}\right)}\left(\|w\|_{L_{T}^{1}\left(\dot{H}^{s}\right)}+\left\|\nabla V_{0}\right\|_{\dot{H}^{s}}+\left\|V_{1}\right\|_{\dot{H}^{s}}\right)
$$

Set

$$
z=\mathscr{B}_{T}(u, w)+\mathscr{L}_{T}(u)=\int_{0}^{t} e^{(t-\tau) \Delta} \operatorname{div}\left(u \nabla S\left(w, V_{0}, V_{1}\right)\right)(\tau) d \tau
$$


Proposition 2.1 provides

$$
\|z\|_{\tilde{L}_{T}^{1}\left(\dot{H}^{s}\right)} \leq C\left\|\operatorname{div}\left(u \nabla S\left(w, V_{0}, V_{1}\right)\right)\right\|_{\tilde{L}_{T}^{1}\left(\dot{H}^{s-2}\right)}
$$

hence

$$
\|z\|_{\tilde{L}_{T}^{1}\left(\dot{H}^{s}\right)} \leq C\left\|u \nabla S\left(w, V_{0}, V_{1}\right)\right\|_{\tilde{L}_{T}^{1}\left(\dot{H}^{s-1}\right)}
$$

Since $-n / 2<s<n / 2$, the product is continuous from $\tilde{L}_{T}^{1}\left(\dot{H}^{s}\right) \times \tilde{L}_{T}^{\infty}\left(\dot{H}^{s}\right)$ to $\tilde{L}_{T}^{1}\left(\dot{H}^{2 s-n / 2}\right)=$ $\tilde{L}_{T}^{1}\left(\dot{H}^{s-1}\right)$. See for instance [1] pages 90 and 98 or use Bony's decomposition. With 17, this implies that

and with 10

$$
\|z\|_{\tilde{L}_{T}^{1}\left(\dot{H}^{s}\right)} \leq C\|u\|_{\tilde{L}_{T}^{1}\left(\dot{H}^{s}\right)}\left\|\nabla S\left(w, V_{0}, V_{1}\right)\right\|_{\tilde{L}_{T}^{\infty}\left(\dot{H}^{s}\right)}
$$

$$
\|z\|_{\tilde{L}_{T}^{1}\left(\dot{H}^{s}\right)} \leq C\|u\|_{\tilde{L}_{T}^{1}\left(\dot{H}^{s}\right)}\left(\|w\|_{\tilde{L}_{T}^{1}\left(\dot{H}^{s}\right)}+\left\|\nabla V_{0}\right\|_{\dot{H}^{s}}+\left\|V_{1}\right\|_{\dot{H}^{s}}\right)
$$

Global existence and uniqueness in theorem 3.2 is a consequence of lemmas 3.1 and 3.2 by restricting to small data, i.e $\left\|\nabla V_{0}\right\|_{\dot{H}^{s}}+\left\|V_{1}\right\|_{\dot{H}^{s}}<1 / C_{1}$ and $\left\|u_{0}\right\|_{\dot{H}^{s-2}}<\left[1-C_{1}\left(\left\|\nabla V_{0}\right\|_{\dot{H}^{s}}+\right.\right.$ $\left.\left.\left\|V_{1}\right\|_{\dot{H}^{s}}\right)\right]^{2} /\left(4 C_{0} C_{2}\right)$. The local existence and uniqueness part is a consequence of the same lemmas once $\lim _{t \rightarrow 0}\left\|e^{\tau \Delta} u_{0}\right\|_{E_{t}}=0$ is proved. Recalling the assumption $u_{0} \in \dot{H}^{s}$, this follows from the fact that $e^{\tau \Delta} u_{0} \in L^{1}\left([0, t], \dot{H}^{s}(\mathbb{R})\right)$ (see proposition 2.1) and the inequality (see [1] p.98) $\left\|e^{\tau \Delta} u_{0}\right\|_{E_{t}} \leq$ $\left\|e^{\tau \Delta} u_{0}\right\|_{L^{1}\left([0, t], \dot{H}^{s}(\mathbb{R})\right)} \rightarrow 0$ when $t \rightarrow 0$.

Remark 3.1. The proof of theorem 3.1 extends to the Debye type system (see [3], [Y] ): $\partial_{t} u_{j}-\Delta u_{j}=$ $\operatorname{div}\left(\beta_{j} u_{j} \nabla V\right), \partial_{t t} V-\Delta V=\sum_{k} \alpha_{k} u_{k}, u_{j}(0)=u_{j, 0}, V(0)=V_{0}, V_{t}(0)=V_{1}$ with $\left(\alpha_{j}, \beta_{j}\right) \in \mathbb{R}^{2}$ $(1 \leq j \leq m)$ given

\section{Existence and uniqueness in the case $n=1$.}

Until the end of the paper, $n=1$. We still denote by $S\left(u, V_{0}, V_{1}\right) \in C^{0}\left(0, T, \mathscr{S}^{\prime}\left(\mathbb{R}^{n}\right)\right)$ the unique solution of the wave equation 2 , 4, and $\mathscr{B}_{T}(u, w)$ and $\mathscr{L}_{T}(u)$ are still formally defined by formulas 12 and 13 . The notation $L_{x}^{p}$ stands for $L^{p}\left(\mathbb{R}_{x}\right)$. Last, $\nabla=\operatorname{div}=\partial_{x}$.

As a building block in the proof of the existence theorem 4.1 below, we first establish a $L^{1}$ estimate for solutions of equations 5, 6 (lemma 4.3). We begin with two simple trace-lemmas. For $y \in \mathbb{R}$, set $\left.D_{T}(y)=\right] 0, T[\times] y, y+1\left[\right.$ and $\bar{D}_{T}(y)=[0, T] \times[y, y+1]$.

Lemma 4.1. Let $T>0, y \in \mathbb{R}$. There exists $C>0$ such that, for any $y \in \mathbb{R}$ and $f \in C^{1}\left(\bar{D}_{T}(y)\right)$, we have

$$
\|f(., y)\|_{L^{2}(0, T)}+\|f(., y+1)\|_{L^{2}(0, T)} \leq C\|f\|_{L^{2}\left(0, T, H^{1}(] y, y+1[)\right)}
$$

Proof. We only prove inequality

$$
\int_{0}^{T}|f(\tau, y)|^{2} d \tau \leq C\|f\|_{L^{2}\left(0, T, H^{1}(] y, y+1[)\right)}^{2}
$$

Let $\phi \in C^{1}\left(\bar{D}_{T}(0)\right)$ with $\phi(t, x)=1$ for $(t, x) \in[0, T] \times[0,1 / 4]$ and $\phi(t, x)=0$ for $(t, x) \in[0, T] \times$ $[3 / 4,1]$. For $y \in \mathbb{R}$ fixed, define $\phi^{y} \in C^{1}\left(\bar{D}_{T}(y)\right)$ by $\phi^{y}(t, x)=\phi(t, x-y)$. Let $f \in C^{1}\left(\bar{D}_{T}(y)\right)$. We have

$$
\int_{0}^{T}|f|^{2}(\tau, y) d \tau=\int_{0}^{T}\left|\phi^{y} f\right|^{2}(\tau, y) d \tau \leq \int_{0}^{T} \int_{y}^{y+1} 2\left|\phi^{y} f\left(\phi^{y} f\right)_{x}\right|(\tau, s) d s d \tau
$$




$$
\leq 2\left\|\phi^{y}\right\|_{W^{1, \infty}(D(y))}^{2}\|f\|_{L^{2}\left(0, T, H^{1}(] y, y+1[)\right)}^{2}
$$

Since $\left\|\phi^{y}\right\|_{W^{1, \infty}(D(y))}=\|\phi\|_{W^{1, \infty}(D(0))}$, we get inequality 19.

Let $y \in \mathbb{R}$. By lemma 4.1, we can define two continuous trace-operators $\gamma_{y+1}^{-}$and $\gamma_{y}^{+}$: $L^{2}\left(0, T, H^{1}(\mathbb{R})\right) \rightarrow L^{2}(0, T)$ by $\gamma_{y}^{+}(f)(\tau)=f(\tau, y)$ and $\gamma_{y+1}^{-}(f)(\tau)=f(\tau, y+1)$ for any $f \in$ $C^{1}\left(\bar{D}_{T}(y)\right)$. For future reference, notice that, for any $f \in L^{2}(] 0, T\left[, H^{1}(] y, y+1[)\right)$, we have

$$
\begin{aligned}
& \left\|\gamma_{y+1}^{-}(f)\right\|_{L^{1}(0, T)}+\left\|\gamma_{y}^{+}(f)\right\|_{L^{1}(0, T)} \\
& \leq \sqrt{T}\left(\left\|\gamma_{y+1}^{-}(f)\right\|_{L^{2}(0, T)}+\left\|\gamma_{y}^{+}(f)\right\|_{L^{2}(0, T)}\right) \leq C \sqrt{T}\|f\|_{L^{2}\left(0, T, H^{1}(] y, y+1[)\right)}
\end{aligned}
$$

with a constant $C>0$ independant of $y \in \mathbb{R}$.

The second lemma is a consequence of the continuity of the trace functions $\gamma_{y}^{ \pm}$and density arguments. We only prove inequality 23. In the sequel sign denotes the sign function.

Lemma 4.2. Let $T>0$ and $y \in \mathbb{R}$. Let also $f \in L^{2}\left(0, T, H^{2}(\mathbb{R})\right), g \in L^{\infty}\left(0, T, H^{2}(\mathbb{R})\right)$ and $\phi \in L^{2}\left(0, T, H^{1}(\mathbb{R})\right)$. Then, for any $(y, z) \in \mathbb{R}^{2}, y<z$, and any $t \in[0, T]$ we have

a)

$$
\begin{gathered}
\int_{0}^{t} \int_{y}^{z} \phi \partial_{x x} f d x d \tau=-\int_{0}^{t} \int_{y}^{z} \partial_{x} \phi \partial_{x} f d x d \tau+\int_{0}^{t}\left[\gamma_{z}^{-}\left(\phi \partial_{x} f\right)-\gamma_{y}^{+}\left(\phi \partial_{x} f\right)\right] d \tau \\
\int_{0}^{t} \int_{y}^{z} \operatorname{sign}(f) \partial_{x}\left(f \partial_{x} g\right) d x d \tau=\int_{0}^{t}\left[\gamma_{z}^{-}\left(|f| \partial_{x} g\right)-\gamma_{y}^{+}\left(|f| \partial_{x} g\right)\right] d \tau
\end{gathered}
$$

b) Let $A \in L^{\infty}\left(0, T, H^{1}(\mathbb{R})\right)$. Then $\left|\gamma_{y}^{ \pm}(A f)\right|(\tau) \leq\|A\|_{L^{\infty}\left(0, T, H^{1}(] y, y+1[)\right)}\left|\gamma_{y}^{ \pm}(f)\right|(\tau)$ for almost every $\tau \in[0, T]$.

Proof. (of 23). We first prove that, for any $(F, G) \in L_{T}^{2}\left(H^{1}\right) \times L_{T}^{\infty}\left(H^{2}\right)$, we have

$$
\int_{0}^{t} \int_{y}^{z} \partial_{x}\left(F \partial_{x} G\right) d x d \tau=\int_{0}^{t}\left[\gamma_{z}^{-}\left(F \partial_{x} G\right)-\gamma_{y}^{+}\left(F \partial_{x} G\right)\right] d \tau
$$

The case $(F, G) \in C^{\infty}([0, T] \times \mathbb{R})^{2}$ is obvious. Since both sides of 24 are continuous with respect to $(F, G) \in L_{T}^{2}\left(H^{1}\right) \times L_{T}^{\infty}\left(H^{2}\right)$, owing to the fact that $H^{1}(\mathbb{R})$ is a Banach algebra and the continuity 21 of the trace functions, we get equality 24 by density. Now, for $f \in L^{2}\left(0, T, H^{1}(\mathbb{R})\right), g \in$ $L^{\infty}\left(0, T, H^{2}(\mathbb{R})\right)$, we have $|f| \in L^{2}\left(0, T, H^{1}(\mathbb{R})\right)$ and $\partial_{x}\left(f \partial_{x} g\right) s g(f)=\partial_{x}\left(|f| \partial_{x} g\right)$. Therefore, equality 23 follows from equality 24 with $F=|f|$ and $G=g$.

We are ready to prove our main $L^{1}$ lemma.

Lemma 4.3. Let $T>0, V_{0} \in H^{2}(\mathbb{R}), V_{1} \in H^{1}(\mathbb{R}), u_{0} \in H^{1}(\mathbb{R}) \cap L^{1}(\mathbb{R})$ and $u \in L_{T}^{2}\left(H^{2}\right) \cap$ $H_{T}^{1}\left(L^{2}\right) \cap C^{0}\left([0, T], H^{1}\right)$. Assume that $S\left(u, V_{0}, V_{1}\right) \in C^{0}\left([0, T], H^{2}\right) \cap C^{1}\left([0, T], H^{1}\right)$ and assume that function $\left(u, S\left(u, V_{0}, V_{1}\right)\right)$ is a solution of equations 5 , 6. i.e

$$
u(t)=e^{t \Delta} u_{0}+\int_{0}^{t} e^{(t-\tau) \Delta} d i v\left(u \nabla S\left(u, V_{0}, V_{1}\right)\right)(\tau) d \tau
$$

Then $u \in C^{0}\left([0, T], L^{1}(\mathbb{R})\right)$ and $\|u(t)\|_{L^{1}} \leq\left\|u_{0}\right\|_{L^{1}}$, for any $t \in[0, T]$. Moreover, when $\pm u_{0} \geq 0$, we have $\pm u \geq 0$ and $\|u(t)\|_{L^{1}}=\left\|u_{0}\right\|_{L^{1}}$. 
Proof. Let $(y, z) \in \mathbb{R}^{2}$ with $y<z$, and $t \in[0, T]$. Since $u \in L_{T}^{2}\left(H^{2}\right)$ and $S\left(u, V_{0}, V_{1}\right) \in$ $C^{0}\left([0, T], H^{2}\right)$, we can apply formula 23 with $f=u, g=S\left(u, V_{0}, V_{1}\right)$. Hence, multiplying 5 by $\operatorname{sign}(u)$ and integrating on $[0, T] \times[y, z]$, we obtain

$$
\begin{aligned}
& \int_{y}^{z}|u|(t, x) d x=\left\|u_{0}\right\|_{L^{1}([y, z])}+\int_{0}^{t}\left[\gamma_{z}^{-}\left(|u| \partial_{x} S\right)-\gamma_{y}^{+}\left(|u| \partial_{x} S\right)\right] d \tau+\int_{0}^{t} \int_{y}^{z} \operatorname{sign}(u) \Delta u d x d \tau \\
& \leq\left\|u_{0}\right\|_{L^{1}([y, z])}+\left\|\gamma_{z}^{-}\left(|u| \partial_{x} S\right)\right\|_{L^{1}([0, T])}+\left\|\gamma_{y}^{+}\left(|u| \partial_{x} S\right)\right\|_{L^{1}([0, T])}+\sup _{t \in[0, T]}\left(\int_{0}^{t} \int_{y}^{z} \operatorname{sign}(u) \Delta u d x d \tau\right)
\end{aligned}
$$

We majorize the last three terms in inequality 25, Using the inequality 21, we get

$$
\left\|\gamma_{z}^{-}\left(|u| \partial_{x} S\right)\right\|_{L^{1}([0, T])}+\left\|\gamma_{y}^{+}\left(|u| \partial_{x} S\right)\right\|_{L^{1}([0, T])} \leq C \sqrt{T}\left\||u| \partial_{x} S\right\|_{L^{2}\left(0, T, H^{1}(] y, y+1[\cup] z-1, z[)\right)}
$$

Hence, 26 and $|u| \partial_{x} S \in L^{2}\left(0, T, H^{1}(\mathbb{R})\right)$ implies that

$$
\lim _{i n f(|y|,|z|) \rightarrow \infty}\left(\left\|\gamma_{z}^{-}\left(|u| \partial_{x} S\right)\right\|_{L^{1}([0, T])}+\left\|\gamma_{y}^{+}\left(|u| \partial_{x} S\right)\right\|_{L^{1}([0, T])}\right)=0
$$

We next prove that

$$
\limsup _{\inf (|y|,|z|) \rightarrow \infty}\left(\sup _{t \in[0, T]} \int_{0}^{t} \int_{y}^{z} \operatorname{sign}(u) \Delta u d x d \tau\right) \leq 0
$$

Set $h_{\epsilon}(x)=x / \sqrt{x^{2}+\epsilon}(x \in \mathbb{R}, \epsilon>0)$. Due to $\Delta u \in L^{2}([0, T] \times \mathbb{R}) \hookrightarrow L_{l o c}^{1}([0, T] \times \mathbb{R}),\left\|h_{\epsilon}\right\|_{L^{\infty}} \leq 1$ and Lebesgue theorem, we have

$$
\int_{0}^{t} \int_{y}^{z} \operatorname{sign}(u) \Delta u d x d \tau=\lim _{\epsilon \rightarrow 0} \int_{0}^{t} \int_{y}^{z} h_{\epsilon}(u) \Delta u d x d \tau
$$

Using 22 with $f=u \in L_{T}^{2}\left(H^{2}\right), \phi=h_{\epsilon}(u) \in L_{T}^{2}\left(H^{1}\right)$, majorizing, and appealing to lemma $\left.4.2 \mathrm{~b}\right)$, we obtain an $(\epsilon, t)$-independent estimate

$$
\begin{aligned}
\int_{0}^{t} \int_{y}^{z} \Delta u h_{\epsilon}(u) d x d \tau & =-\int_{0}^{t} \int_{y}^{z}|\nabla u|^{2} h_{\epsilon}^{\prime}(u) d x d \tau+\int_{0}^{t}\left[\gamma_{z}^{-}\left(h_{\epsilon}(u) \nabla u\right)-\gamma_{y}^{+}\left(h_{\epsilon}(u) \nabla u\right)\right] d \tau \\
& \leq \int_{0}^{T}\left[\left|\gamma_{z}^{-}(\nabla u)\right|+\left|\gamma_{y}^{+}(\nabla u)\right|\right] d \tau
\end{aligned}
$$

Appealing to 21, we deduce from 30 and 29 that

$$
\sup _{t \in[0, T]}\left(\int_{0}^{t} \int_{y}^{z} \operatorname{sign}(u) \Delta u(\tau, x) d x d \tau\right) \leq C \sqrt{T}\|\nabla u\|_{L^{2}\left(0, T, H^{1}(] y, y+1[\cup] z-1, z[)\right.}
$$

Hence, inequality 28 follows from 31 and $u \in L^{2}\left(0, T, H^{2}(\mathbb{R})\right)$. Set $y=-z$ and let $z \rightarrow+\infty$ in 25. Using 27, 28 and the monotone convergence theorem, we get $\|u(t)\|_{L^{1}} \leq\left\|u_{0}\right\|_{L^{1}}$ for $t \in[0, T]$. Replacing the sign function by the negative or positive part functions $(.)^{ \pm}$, or the constant function 1 , the same argument provides $\left\|(u)^{ \pm}(t)\right\|_{L^{1}} \leq\left\|\left(u_{0}\right)^{ \pm}\right\|_{L^{1}}$ and $\int_{\mathbb{R}} u(t, x) d x=\int_{\mathbb{R}} u_{0}(x) d x$. In the particular case $\pm u_{0} \geq 0$, we recover $\pm u(t) \geq 0$ and $\|u(t)\|_{L^{1}}=\left\|u_{0}\right\|_{L^{1}}$ for any $t \in[0, T]$.

Finally, appealing to 25, [27, [28 and $u_{0} \in L^{1}$, we have $\sup _{\tau \in[0, T]}\|u(\tau)\|_{L^{1}(]-\infty,-x[\cup] x,+\infty[)} \rightarrow 0$ when $x \rightarrow+\infty$. Hence, noticing that $u \in C^{0}\left(0, T, L^{2}(\mathbb{R})\right) \hookrightarrow C^{0}\left(0, T, L_{\text {loc }}^{1}(\mathbb{R})\right)$, we easily obtain $u \in C^{0}\left(0, T, L^{1}(\mathbb{R})\right)$ 
It follows that

Theorem 4.1. Let $T>0, V_{0} \in H^{2}(\mathbb{R}), V_{1} \in H^{1}(\mathbb{R})$ and $u_{0} \in H^{1}(\mathbb{R}) \cap L^{1}(\mathbb{R})$. The problem find $u \in L_{T}^{2}\left(H^{1}\right)$ such that

$$
u(t)=e^{t \Delta} u_{0}+\int_{0}^{t} e^{(t-\tau) \Delta} d i v\left(u \nabla S\left(u, V_{0}, V_{1}\right)\right)(\tau) d \tau
$$

admits exactly one solution. Moreover, $u \in L_{T}^{2}\left(H^{2}\right) \cap H_{T}^{1}\left(L^{2}\right) \cap C^{0}\left([0, T], H^{1} \cap L^{1}\right)$ and $\|u(t)\|_{L_{x}^{1}} \leq$ $\left\|u_{0}\right\|_{L^{1}}$. Last, when $\pm u_{0} \geq 0$, we have $\pm u \geq 0$ and $\|u(t)\|_{L^{1}}=\left\|u_{0}\right\|_{L^{1}}, 0 \leq t \leq T$.

Proof. Step 1 (local existence). For any $0<T<1, u \in L_{T}^{2}\left(H^{1}\right)$ and $w \in L_{T}^{2}\left(H^{1}\right)$, proposition 2.1 provides

$$
\begin{aligned}
\left\|\mathscr{B}_{T}(u, w)+\mathscr{L}_{T}(u)\right\|_{L_{T}^{2}\left(H^{1}\right)} & \leq C\left\|\operatorname{div}\left(u \nabla S\left(w, V_{0}, V_{1}\right)\right)\right\|_{L_{T}^{1}\left(L^{2}\right)} \\
& \leq C\left\|u \nabla S\left(w, V_{0}, V_{1}\right)\right\|_{L_{T}^{1}\left(H^{1}\right)} \\
& \leq C\|u\|_{L_{T}^{2}\left(H^{1}\right)}\left\|\nabla S\left(w, V_{0}, V_{1}\right)\right\|_{L_{T}^{2}\left(H^{1}\right)}
\end{aligned}
$$

Note that

$$
S\left(w, V_{0}, V_{1}\right)(t, x)=\int_{0}^{t} \int_{x-(t-\tau)}^{x+(t-\tau)} w(\tau, \xi) d \xi d \tau+\frac{1}{2}\left(V_{0}(x+t)+V_{0}(x-t)+\int_{x-t}^{x+t} V_{1}(s) d s\right)
$$

Assume first that $V_{0}, V_{1}$ and $u$ are infinitely differentiable. We have

$$
\begin{aligned}
\nabla S\left(w, V_{0}, V_{1}\right)(t, x)= & \int_{0}^{t}(w(\tau, x+(t-\tau))-w(\tau, x-(t-\tau)) d \tau \\
& +\frac{1}{2}\left(V_{0}^{\prime}(x+t)+V_{0}^{\prime}(x-t)+V_{1}(x+t)-V_{1}(x-t)\right)
\end{aligned}
$$

Arguing by density, the formula 34 holds for the distributional derivative $\nabla S$ under the assumptions of theorem 4.1, and we have

$$
\left\|\nabla S\left(w, V_{0}, V_{1}\right)\right\|_{L_{T}^{2}\left(H^{1}\right)} \leq C \sqrt{T}\left(\|w\|_{L_{T}^{2}\left(H^{1}\right)}+\left\|V_{0}^{\prime}\right\|_{H^{1}}+\left\|V_{1}\right\|_{H^{1}}\right)
$$

From 32 and 35 we deduce that

$$
\left\|\mathscr{B}_{T}(u, w)+\mathscr{L}_{T}(u)\right\|_{L_{T}^{2}\left(H^{1}\right)} \leq C \sqrt{T}\|u\|_{L_{T}^{2}\left(H^{1}\right)}\left(\|w\|_{L_{T}^{2}\left(H^{1}\right)}+\left\|V_{0}^{\prime}\right\|_{H^{1}}+\left\|V_{1}\right\|_{H^{1}}\right)
$$

Finally, notice that

$$
\left\|e^{t \Delta} u_{0}\right\|_{L_{T}^{2}\left(H^{1}\right)} \leq\left\|u_{0}\right\|_{L^{2}}
$$

Hence, for $T>0$ small enough, the existence and uniqueness of a solution $u \in L_{T}^{2}\left(H^{1}\right)$ follows from lemma 3.1, inequalities 36 and 37 .

Next, since $u \in L_{T}^{2}\left(H^{1}\right), V_{0} \in H^{2}$ and $V_{1} \in H^{1}$ we get

$$
S\left(u, V_{0}, V_{1}\right) \in C^{0}\left([0, T], H^{2}\right) \cap C^{1}\left([0, T], H^{1}\right)
$$

Therefore $\operatorname{div}\left(u \nabla S\left(u, V_{0}, V_{1}\right)\right) \in L_{T}^{2}\left(L^{2}\right)$. Due to $u_{0} \in H^{1}$, equation $\partial_{t} u-\Delta u=\operatorname{div}\left(u \nabla S\left(u, V_{0}, V_{1}\right)\right)$, proposition 2.1 (and by interpolation), we thus obtain

$$
u \in L_{T}^{2}\left(H^{2}\right) \cap H_{T}^{1}\left(L^{2}\right) \cap C^{0}\left([0, T], H^{1}\right)
$$


Step 2 (global existence). Let $T^{*}>0$, the maximal time of existence of a mild solution endowed with the properties [38, 39, In particular, $u \in C^{0}\left([0, T], L^{1}(\mathbb{R})\right)$ and $\|u(t)\|_{L_{x}^{1}} \leq\left\|u_{0}\right\|_{L^{1}}$ for $0 \leq t \leq$ $T<T^{*}$ (see lemma 4.3). In order to prove that $T^{*}=\infty$, and due to 38, 39 and lemma 4.3, we essentially have to find an a priori estimate on $\|u\|_{L_{T}^{2}\left(H^{1}\right)}$ for $0<T<T^{*}$. We multiply equation 5 by $u$ and integrate we respect to $x$. Appealing to 38 and 39 , we get

$$
\begin{aligned}
\frac{1}{2} \frac{d}{d t}\|u\|_{L_{x}^{2}}^{2}+\int_{\mathbb{R}}\left|\partial_{x} u\right|^{2} d x & \leq\left|\int_{\mathbb{R}} u \partial_{x} u \partial_{x} S d x\right| \\
& \leq\|u\|_{L_{x}^{4}}\left\|\partial_{x} u\right\|_{L_{x}^{2}}\left\|\partial_{x} S\right\|_{L_{x}^{4}}
\end{aligned}
$$

We now estimate $\|u\|_{L_{x}^{4}}$ and $\left\|\partial_{x} S\right\|_{L_{x}^{4}}$. By Gagliardo-Nirenberg inequalities and the $\mathrm{L}^{1}$ properties of $u$, we have

$$
\|u\|_{L_{x}^{4}}^{4} \leq C\|u\|_{L_{x}^{1}}^{2}\left\|\partial_{x} u\right\|_{L_{x}^{2}}^{2} \leq C\left\|u_{0}\right\|_{L^{1}}^{2}\left\|\partial_{x} u\right\|_{L_{x}^{2}}^{2}
$$

Using equation 34 with $w=u$, we obtain for any $0<t<T$

$$
\left\|\partial_{x} S(t)\right\|_{L^{4}} \leq C\left(\|u\|_{L^{1}\left(0, t, L^{4}\right)}+\left\|V_{0}^{\prime}\right\|_{L^{4}}+\left\|V_{1}\right\|_{L^{4}}\right)
$$

Invoking inequality 41, this implies that

$$
\begin{aligned}
\left\|\partial_{x} S(t)\right\|_{L^{4}} & \leq C\left(\left\|u_{0}\right\|_{L^{1}}^{1 / 2} \int_{0}^{t}\left\|\partial_{x} u(\tau)\right\|_{L^{2}}^{1 / 2} d \tau+\left\|V_{0}^{\prime}\right\|_{L^{4}}+\left\|V_{1}\right\|_{L^{4}}\right) \\
& \leq C\left(\left\|u_{0}\right\|_{L^{1}}^{1 / 2}\left\|\partial_{x} u\right\|_{L^{2}(] 0, T[\times \mathbb{R})}^{1 / 2} t^{3 / 4}+\left\|V_{0}^{\prime}\right\|_{L^{4}}+\left\|V_{1}\right\|_{L^{4}}\right)
\end{aligned}
$$

Therefore, 40, 41, 43 and injection $H^{1}(\mathbb{R}) \hookrightarrow L^{4}(\mathbb{R})$ provide

$$
\begin{aligned}
\frac{1}{2} \frac{d}{d t}\|u\|_{L_{x}^{2}}^{2}+\left\|\partial_{x} u(t)\right\|_{L_{x}^{2}}^{2} & \leq C\left\|u_{0}\right\|_{L^{1}}^{1 / 2}\left\|\partial_{x} u(t)\right\|_{L^{2}}^{3 / 2}\left(\left\|u_{0}\right\|_{L^{1}}^{1 / 2}\left\|\partial_{x} u\right\|_{L^{2}(] 0, T[\times \mathbb{R})}^{1 / 2} t^{3 / 4}+\left\|V_{0}^{\prime}\right\|_{L^{4}}+\left\|V_{1}\right\|_{L^{4}}\right) \\
& \leq \eta\left\|u_{0}\right\|_{L^{1}}^{2 / 3}\left\|\partial_{x} u(t)\right\|_{L^{2}}^{2} \\
& +C_{\eta}\left(t^{3}\left\|u_{0}\right\|_{L^{1}}^{2}\left\|\partial_{x} u\right\|_{L^{2}(] 0, T[\times \mathbb{R})}^{2}+\left\|V_{0}\right\|_{H^{2}}^{4}+\left\|V_{1}\right\|_{H^{1}}^{4}\right)
\end{aligned}
$$

Take $\eta$ such that $0<\eta\left\|u_{0}\right\|_{L^{1}}^{2 / 3} \leq 1 / 2$. Hiding the term $\eta\left\|u_{0}\right\|_{L^{1}}^{2 / 3}\left\|\partial_{x} u(t)\right\|_{L^{2}}^{2}$ in the left hand side of 44, setting $z(t)=\|u(t)\|_{L^{2}}^{2}+\int_{0}^{t}\left\|\partial_{x} u(\tau)\right\|_{L^{2}}^{2} d \tau$ and using the Gronwall inequality we obtain the required a priori estimate on $\|u\|_{L_{T}^{2}\left(H^{1}\right)}$. The end of the proof is standard and we omit further details.

\section{References}

[1] H.Bahouri, J. Y. Chemin and R. Danchin, Fourier Analysis and Nonlinear Partial Differential Equations, vol. 343, Springer-Verlag Berlin Heidelberg, 2011.

[2] P. Biler and J. Dolbeault, Long Time Behavior of Solutions to Nernst-Planck and DebyeHuckel Drift-Diffusion Systems, Ann. Henri Poincaré 1 (2000), pp.461-472

[3] P. Biler, W. Hebisch and T. Nadzieja, The Debye system, existence and time behavior of solutions, Nonlinear Analysis, Theory, Methods and Applications, Vol. 23, No. 9. pp. 11891209, 1994. 
[4] J.M. Chemin, Remarques sur l'existence globale pour l'équation de Navier-Stokes incompressible, SIAM Journal of Math. Anal. 23 (1992) pp.20-28.

[5] P. Constantin and G. Seregin, Global regularity of solutions of coupled Navier-Stokes equations and nonlinear Fokker Planck equations, Discrete and continuous dynamical systems, 26 (4), pp. 1185-1196 (2010).

[6] T. Kato, Strong $L^{p}$ solutions of the Navier-Stokes equations in $\mathbb{R}^{m}$ with applications to weak solutions, Math. Zeit, 187(1984), pp.471-480.

[7] A. Krzywicki and T. Nadzieja, A nonstationary problem in the theory of electrolytes, Quaterly Applied Math. 50, 105-107, 1992.

[8] P.G. Lemarié-Rieusset, Recents developments in the Navier-Stokes problem, Chapman et Hall, 2002.

[9] F.B. Weissler, The Navier-Stokes initial valued problem in $L^{p}$, Arch. Rat. Mech. and Anal. 74 (1981) pp. 219-230.

[10] M. Winkler, Finite-time blow-up in the higher-dimensional parabolic-parabolic Keller-Segel system, Journal de Mathématiques Pures et Appliquées, Volume 100, Issue 5, November 2013, pp. 748-767 